Bailo, G.LLitigación sobre polinizadores (el derecho según las abejas)Derecho y Ciencias Sociales. Mayo-Octubre 2019 . № 21.

Pgs 263-283. ISNN 1852-2971. Instituto de Cultura Jurídica y Maestría en Sociología Jurídica. FCJ y S. UNLP

\title{
Litigación sobre polinizadores (el derecho según las abejas)
}

Litigation on pollinators (the law according to bees)

Gonzalo L. Bailo•

\section{Resumen}

La presencia de los polinizadores es esencial para la biodiversidad y la producción de alimentos. La exposición a múltiples factores de estrés está provocando la pérdida de numerosas colonias de abejas melíferas y de distintos polinizadores silvestres. El objeto del trabajo es analizar el estado actual de la litigación respecto a algunos de estos factores de estrés. Se examinan sucesivamente casos que involucran neonicotinoides, sulfoxaflor, fipronil, glifosato, ácido 2,4-diclorofenoxiacético y dimetoato. Se concluye que la litigación clásica sobre las abejas, centrada en problemas de propiedad y de responsabilidad civil por daños, está virando hacia una litigación sobre polinizadores, que intenta proteger la biodiversidad y la alimentación.

Palabras clave: Polinizadores; abejas; litigación sobre polinizadores; pesticidas; diversidad biológica

\begin{abstract}
The presence of pollinators is essential for biodiversity and food production. Exposure to multiple stress factors is causing the loss of numerous colonies of honey bees and different wild pollinators. The purpose of the work is to analyze the current state of litigation regarding some of those stress factors. It is examined cases involving neonicotinoids, sulfoxaflor, fipronil, glyphosate, 2,4-dichlorophenoxyacetic acid and dimethoate. It is concluded that the classic litigation on bees, centered on problems of property and liability for damages, is turning towards a litigation on pollinators, which tries to protect biodiversity and food.
\end{abstract}

Key-words: Pollinators; bees; litigation on pollinators; pesticides; biological diversity

\footnotetext{
- Becario Conicet (Argentina). Docente de Derecho Civil II (Obligaciones) y de Derecho de Daños (FCJS-Uiversidad Nacional del Litoral - Santa Fé- Argentina). Centro de Investigaciones en Derecho (FCJS-UNL). Correo electrónico: gbailo@fcjs.unl.edu.ar Orcid: http://orcid.org/0000-0002-7395-2630
}

Recibido: 5/2/2019. Publicable con correcciones: 14/7/2019 
Bailo, G.LLitigación sobre polinizadores (el derecho según las abejas)Derecho y Ciencias Sociales. Mayo-Octubre 2019 . № 21. Pgs 263-283. ISNN 1852-2971. Instituto de Cultura Jurídica y Maestría en Sociología Jurídica. FCJ y S. UNLP

\section{Litigación sobre polinizadores (el derecho según las abejas)}

Gonzalo L. Bailo

\section{Introducción. Abejas y polinizadores en el derecho.}

Existen en el planeta más de 25.000 especies de abejas (Gould, 2015). La presencia de estas especies y de otros polinizadores es esencial para la biodiversidad y la producción de alimentos. La polinización es un servicio de regulación del ecosistema de gran importancia para la naturaleza, la agricultura y el bienestar humano. Sin embargo, la exposición crónica de las abejas a múltiples factores de estrés está llevando a la pérdida de colonias de abejas melíferas y de distintos polinizadores silvestres (Potts et al, 2010; Goulson et al, 2015).

Con el objeto de sensibilizar a la opinión pública y construir cursos de acción para la protección de las abejas, la Asamblea General de la ONU (2017) estableció al 20 de mayo como "Día Mundial de las Abejas". En el campo internacional, los principales cursos de acción en materia de protección de polinizadores se concentran en el Convenio de Diversidad Biológica (CDB) y en la Organización de las Naciones Unidas para la Alimentación y la Agricultura (FAO).

En el ámbito del CDB, la Tercera Conferencia de las Partes (COP) reconoció en 1996 la importancia de los polinizadores y la necesidad de abordar las causas de su declive poblacional (Decisión III/11). En el año 2000, la Quinta Conferencia adoptó la Iniciativa Internacional sobre Polinizadores (International Pollinator Initiative), que funciona en el programa de trabajo de biodiversidad en la agricultura, y que es coordinada por FAO (Decisión V/5). En el año 2002, la Sexta Conferencia adoptó el Plan de Acción de la Iniciativa Internacional sobre Polinizadores (Decisión VI/5), aprobó la Iniciativa Mundial Sobre Taxonomía (Decisión VI/8) y delineó los respectivos mecanismos de financiación (Decisión VI/17). Los polinizadores han sido también objeto de tratamiento en la Novena Conferencia (Decisión IX/1), en la Décima Conferencia (Decisiones X/24 y X/34) y en la Décimo Tercera Conferencia (Decisión XIII/15). En esta última Conferencia, la Secretaría del CDB y la FAO prepararon el Plan Actualizado de Acción 20182030 para la Iniciativa Internacional sobre la Conservación y el Uso Sostenible de los Polinizadores.

\footnotetext{
${ }^{1}$ En la Resolución, la ONU reconoce la importancia de los polinizadores para la seguridad alimentaria y la salud del ecosistema, y reseña algunos de los factores que amenazan su hábitat, salud y desarrollo. La protección de los polinizadores también tiene puntos de conexión con la Agenda 2030 para el Desarrollo Sostenible, especialmente con el objetivo "Hambre Cero" por su importancia para la alimentación y la nutrición.
} 
En el ámbito de la FAO, se destaca la Acción Mundial sobre Servicios de Polinización para una Agricultura Sostenible y el Proyecto sobre conservación y gestión de los polinizadores para la agricultura sostenible a través de un enfoque por ecosistemas.

El Tribunal Permanente de los Pueblos (foro no gubernamental)también se hizo eco del problema de los polinizadores en una sesión sobre corporaciones transnacionales de agroquímicos realizada en diciembre de $2011 .^{2}$

Atendiendo a estos antecedentes, el objeto del trabajo es analizar el estado actual de la litigación sobre los factores de estrés a los que están expuestos los polinizadores. La exposición se estructura de acuerdo a los principales tipos de litigios que aborda la jurisprudencia reciente, abocada casi exclusivamente a los efectos que ciertos tipos de pesticidas producen en las abejas melíferas. Se analizan los casos que involucran neonicotinoides, sulfoxaflor, fipronil, glifosato, ácido 2,4diclorofenoxiacético y dimetoato. Finalmente, se ofrecen algunas conclusiones provisorias.

\section{La litigación y los polinizadores. Un aprendizaje continuo sobre la causalidad}

Karl Von Frisch, el etólogo austríaco que dedicó gran parte de su vidaal estudio de la danza de las abejas, expresó en el prefacio de su obra "The dancing bees" (1955) que emplear aparatos excesivamente sofisticados para examinar fenómenos naturales simples puede hacer que la naturaleza se nos escape fácilmente. Las abejas utilizan la danza para comunicarse. La danza circular (round dance) sirve para que los miembros de la colmena busquen en los alrededores de la colmena (Von Frisch, 1974: 664). La danza del coleteo (tail-wagging dance) se utiliza para misiones a mayores distancias, e indica con precisión no sólo la distancia del objetivo sino también la dirección (Von Frisch, 1974: 665).

El lenguaje de las abejas sirve para comprender mejor la crisis de los polinizadores.Las numerosas señales de alerta que éstos comunican, especialmente a través de sus vuelos, han comenzado a producir cambios en las estrategias de litigaciónde los apicultores, las organizaciones no gubernamentales y las agencias estatales.

En los primeros códigos civiles el vuelo de las abejas era un hecho relevante para establecer la propiedad de los enjambres ${ }^{3}$ y para regular los supuestos de responsabilidad por daños provocados

\footnotetext{
${ }^{2}$ En esta sesión, el Tribunal Permanente denunció que la toxicidad de muchos agroquímicos afecta a distintos animales de forma directa y a otros de forma indirecta a través de la cadena alimentaria. También expresó que los monocultivos, el uso de maquinaria industrial en la agricultura y la aplicación de fertilizantes y pesticidas han producido grandes pérdidas de polinizadores en distintas partes del planeta.

${ }^{3}$ El Código Civil argentino de 1869, por ejemplo, establecía en su artículo 2343 que los enjambres de abejas eran susceptibles de apropiación privada si el propietario de ellos no los reclamare inmediatamente, y expresaba en su artículo 2545 que si las abejas huyen de la colmena y posan en árbol que no sea del propietario de ella, se entiende que vuelven a su libertad natural, si el dueño no fuese en seguimiento de ellas. El artículo siguiente regulaba la hipótesis
} 
por abejas y enjambres a seres humanos o a otros animales. ${ }^{4}$ La litigación actual sobre polinizadores, que está en pleno proceso de exploración de sus propios límites y posibilidades, presenta-al menos- dos aspectos problemáticos recurrentes: a) la necesidad de construir un interés a proteger y una legitimación procesal que supere el mero interés económico de los sujetos privados; y b) la pluralidad de factores causales comprometidos en la declinación de los polinizadores, como cuestión que dificulta la producción de evidencia en los casos concretos. ${ }^{5}$

Estos nuevos litigios revelan la limitada capacidad de los sistemas humanos -especialmente de los jurídicos- para comprender sistemas complejos y multidimensionales. De acuerdo a Goulson et al (2015: 4), muchas veces la falta de evidencia clara de los daños in situ se malinterpreta como evidencia de que los estudios toxicológicos que se realizan sobre abejas individuales no pueden aplicarse a las pérdidas de colonias en el terreno, cuando estoindica sólo ausencia de evidencia.Algunas investigaciones sugieren que, no obstante los vacíos en el conocimiento que se dan en cada caso particular, hay suficiente evidencia acumulada sobre los efectos adversos a largo plazo que pueden tener los pesticidas sobre la salud de las abejas como para reevaluar ciertos riesgos (cfr. Baines et al, 2017).

Por estas razones, cuando los jueces deben resolver los casos sobre polinizadores recurriendoa sistemas jurídicos incompletos y a herramientas de análisis causal que suelen resistirse a trabajar con casos únicos, surgenalgunos desafíos especiales.

Un buen ejemplo de elloes el casoAnklagemyndigheden v. Ditlev Bluhme, fallado en 1998 por el Tribunal de Justicia de la Unión Europea (ECLI:EU:C:1998:584).En el año 1993, Dinamarca había prohibido poseer en la Isla de Læsø abejas que no pertenecieran a la subespecie Apis mellifera mellifera (abeja parda de Læsø). El Estado danés se amparaba en la necesidad de proteger este tipo particular de abejas de una eventual desaparición, argumentando que las abejas pardas de Læsø sólo pueden preservarse en esa isla. Bluhme, un apicultor local que por violar la prohibición fue sometido a proceso penal, alegó en su defensa que las abejas pardas de Læsø no son únicas en su género, que no están bajo situación de amenaza y que el Estado danés, al prohibir la posesión de otras especies de abejas, había violado la prohibición de derecho comunitario de

del enjambre que se posa en terreno ajeno, cercado o cultivado. En ese caso, el Código prescribía que el dueño que lo persiguiese no podía tomarlo sin consentimiento del propietario del terreno. La disposición estaba inspirada en el artículo 620 del Código Civil chileno de Andrés Bello.

${ }^{4}$ El artículo 1124 del Código Civil argentino de 1869 establecía que el propietario de un animal doméstico o feroz, era responsable del daño que cause. No es extraño encontrar sentencias en las que los daños que provocan las abejas son imputados a los dueños de las colmenas. En un caso, por ejemplo, se hizo lugar a la acción de daños interpuesta por el propietario de una yegua que falleció al ser atacada por abejas pertenecientes a colmenas situadas en un predio vecino (AR/JUR/48408/2013).

${ }^{5}$ Para un análisis de algunos fallos clásicos sobre la propiedad de abejas y enjambres véase: Doutt (1959). Para trabajos más recientes véase: Ortiz García (2014) y Jiménez (2018). 
establecer medidas de efecto equivalente a una restricción cuantitativa a la importación. El Estado danés alegó que la medida no estaba referida al acceso a las abejas como mercancías, sino que buscaba la protección de la diversidad biológica, por lo que la normativa nacional no estaba comprendida en el ámbito de aplicación de la previsión comunitaria.

En su sentencia, el Tribunal de Justicia de la Unión Europea concluyó que la prohibición impuesta por Dinamarca constituía una medida de efecto equivalente a una restricción cuantitativa, dado que producía un impacto directo e inmediato sobre los intercambios y podía obstaculizar el comercio entre los Estados, pero que tal medida debía considerarse justificada por razones de protección de la salud y vida de los animales. Como ha apuntado Jacobs (1999: 221) este caso trabaja un conflicto entre normas de libre comercio y normas de protección ambiental (al menos en lo que refiere a la protección de la diversidad biológica). ${ }^{6}$

Puede citarse también un ejemplo de la jurisprudencia francesa. En abril de 2000, durante el período de floración de la colza, algunos apicultores franceses percibieron un aumento significativo de mortalidad de abejas y una disminución correlativa en la actividad de sus colmenas. Presumiendo que ello se debía a la aplicación de fungicidas e insecticidas en los campos decolza linderos, los apicultores, junto con otras organizaciones, demandaron a la cooperativa agrícola que explotaba dichos campos. En el caso, la relación entre las fumigaciones efectuadas y la mortandad de las abejas fue objeto de controversia. Finalmente, en diciembre de 2008, una Corte de Apelaciones de Angers (Francia) condenó a los demandadosa reparar los daños que había provocado a los colmenares del actor (Affaire $n^{\circ}$ 07/01836). A juicio del tribunal, cuando se trata de cuestiones ambientales, el vínculo causal entre la falta y el daño sufrido debe evaluarse teniendo en cuenta el mayor riesgo de causar el daño,como algo inherente al uso de productos fitosanitarios. ${ }^{7}$

Teniendo en cuenta la necesidad de constante aprendizaje causal que demanda la litigación sobre polinizadores, se desarrolla a continuación la jurisprudencia relevante en la materia, que se ha

\footnotetext{
${ }^{6}$ De acuerdo a este autor, el caso es novedoso porque apunta a la protección de la vida de una subespecie de abejas como tal, y porque demuestra el potencial que tiene el CDB para que los Estados tomen medidas de conservación de las especies y de la diversidad biológica en sus territorios.

${ }^{7}$ La Tercera Conferencia de las Partes (COP) ya reconocía en 1996 que para llevar adelante estudios de caso era necesario, entre otras cosas, identificar las causas específicas del declive de polinizadores. De forma correlativa, la Iniciativa Internacional sobre Polinizadores del año 2000 planteó la necesidad de promover acciones coordinadas para monitorear las causas de ese declive y su impacto en los servicios de polinización, como así también de abordar el problema de la falta de información taxonómica. El Plan de Acción de la Iniciativa Internacional sobre Polinizadores, adoptado en la Sexta Conferencia, reconoció la falta de conocimiento que existe sobre las relaciones entre las especies individuales de plantas y sus polinizadores, y cuán específicas se habían demostrado esas relaciones en distintos estudios científicos.
} 
Bailo, G.LLitigación sobre polinizadores (el derecho según las abejas)Derecho y Ciencias Sociales. Mayo-Octubre 2019. № 21. Pgs 263-283. ISNN 1852-2971. Instituto de Cultura Jurídica y Maestría en Sociología Jurídica. FCJ y S. UNLP

concentrado casi exclusivamente en pesticidas que ocasionan elevados índices de mortandadendistintos tipos de abejas melíferas.

\section{La litigación sobre los neonicotinoides}

Los pesticidas son la causa más debatida y controvertida de la mortandad de abejas. Como la mayoría de los pesticidas que se usan en la agricultura son tóxicos para las abejas, su uso tiene un impacto directo en la mortandad de abejas melíferas y silvestres (Pimentel, 1992: 754). En particular, los insecticidas neonicotinoides están fuertemente vinculados con la disminución en las poblaciones de abejas que se han registrado en las últimas décadas.

Según la Autoridad Europea de Seguridad Alimentaria (EFSA) la mayoría de los usos de pesticidas neonicotinoides representan un riesgo tanto para las abejas melíferas como para las silvestres.Algunos estudios demuestran que las abejas no pueden controlar la exposición a los pesticidas neonicotinoides. Así, se ha indicado que las abejas y abejorros tienden a preferir soluciones que contienen imidacloprid y tiametoxam, lo que implica un riesgo considerable para las abejas obreras (Kessler et al, 2015). También se ha apuntado el impacto negativo de los neonicotinoides sobre las abejas reinas melíferas, especialmente en su anatomía reproductiva y fisiológica (Williams et al, 2015).

En 2008, la Oficina Federal Alemana de Protección al Consumidor y Seguridad Alimentaria (BVL) suspendió los registros de ocho productos de tratamiento de semillas de plaguicidas utilizados en aceite de colza y maíz dulce. La medida se basó en informes de apicultores alemanes de la región de Baden-Württemberg, que reportaron la muerte de dos tercios de sus abejas tras la aplicación del pesticida clotianidina (Benjamin, 2008).

En septiembre de 2008, el Ministerio de Trabajo, Salud y Política Social de Italia dictó un decreto que suspendió de forma precautoria las autorizaciones de uso de productos fitosanitarios para tratamiento de semillas que contuvieran las sustancias activas clotianidina, tiametoxam, imidacloprid y fipronil (Gazzetta Ufficiale n. 221 del 20.09.2008). ${ }^{8}$

El 24 de mayo de 2013, la Comisión Europea, a partir del asesoramiento experto de la EFSA, dictó el Reglamento de Ejecución No 485/2013, que modificó el Reglamento de Ejecución (UE) $\mathrm{N}^{\mathrm{o}} 540 / 2011$, y prohibió el uso y la venta de semillas tratadas con productos fitosanitarios que contengan clotianidina, tiametoxam e imidacloprid, con excepción de las semillas usadas en

\footnotetext{
${ }^{8}$ La medida se motivó en una serie de comunicaciones que daban cuenta de decrecimientos poblacionales de abejas en la región de Lombardía y en la región del Piamonte, en algunos informes de agencias públicas, en el principio de precaución del Reglamento (CE) 178/2002, en las medidas que otros países europeos ya habían adoptado sobre prohibición de neonicotinoides y en la protección de la salud pública, animal y medioambiental.
} 
invernaderos.Los grupos empresarios Bayer, Syngenta y BASF, afectados directos por esta medida, demandaron ante el Tribunal General de la Unión Europea la anulación de dichas prohibiciones y restricciones. En mayo de 2018 el Tribunal desestimó las pretensiones. Cabe aclarar que el recurso de BASF prosperó parcialmente, y el Tribunal anuló las medidas que limitan el uso del plaguicida fipronil, por imponerse sin que mediara una evaluación previa de su impacto (ECLI:EU: T:2018:280).

Según el Tribunal General, la Comisión consiguió demostrar que los riesgos justificaban las prohibiciones y restricciones impuestas, habida cuenta de las exigencias relativas a la ausencia de efectos inaceptables de las sustancias activas sobre las abejas.El Tribunal sostuvo que las instituciones pueden adoptar medidas de protección sin necesidad de esperar que la realidad y la gravedad de los riesgos controvertidos queden plenamente demostradas o que se materialicen efectos perjudiciales para la salud. En el mismo sentido, el Tribunal sostuvo que, en la lógica del principio precautorio, la salud pública, la seguridad y el medio ambiente tienen primacía sobre los intereses económicos.

En agosto de 2016, Francia sancionó la Ley para la Recuperación de la Biodiversidad, la Naturaleza y los Paisajes, cuyo artículo 125 modificó la redacción del artículo L253-8 del Código Rural y de Pesca Marítima (Code rural et de la pêche maritime).Esta Ley introdujo la prohibición de usar productos fitosanitarios que contengan sustancias activas de la familia de los neonicotinoides y semillas tratadas con estos productos, a partir del 01 de septiembre de $2018 .^{9}$

La controversia en torno a los efectos de los neonicotinoides en los polinizadores también ha motivado pronunciamientos en la justicia estadounidense. En noviembre de 2016, una Corte de Distrito de California desestimó una demanda de un grupo de apicultores y organizaciones no gubernamentales contra la EPA (Case No. 3:16-cv-00068-WHA). La demanda buscaba la protección de las abejas melíferas y de otros polinizadores de los daños que producen las semillas recubiertas con ciertos insecticidas. Según los demandantes, la práctica de revestir semillas con neonicotinoides tiene un impacto sistemático y catastrófico en las abejas y la industria de la apicultura, dado que el pesticida se distribuye por toda la planta y mata a los insectos por contacto

\footnotetext{
${ }^{9}$ La Ley se motivó en un informe realizado por la Agencia Nacional de Seguridad Sanitaria y en los impactos de los neonicotinoides sobre el medio ambiente -incluidos los polinizadores, la salud pública y la actividad agrícola. Francia ya contaba con importantes antecedentes en el tema. En 1999, el Ministro de Agricultura francés suspendió el uso de imidacloprid en las semillas de girasol y designó un Comité de expertos para examinar su impacto en las abejas. En 2003, este Comité Científico y Técnico emitió un informe, que concluyó que el imidacloprid representa un riesgo significativo para las abejas. En 2004, el Ministro de Agricultura francés suspendió el uso de imidacloprid como tratamiento de semillas para el maíz.
} 
directo y a través de las plantas que ingieren. En la demanda se alegaba que cuando se siembran estas semillas, pueden liberarse pesticidas por desempolvamiento.

La EPA, a través de una guía(Guidance for Inspecting Alleged Cases of Pesticide-Related Bee Incidents), había eximido a las semillas revestidas (y a cualquier polvo resultante de la misma) del registro de la Ley Federal de Insecticidas, Fungicidas y Rodenticidas (FIFRA). ${ }^{10}$ Por esta razón, la demanda apuntaba a la revisión judicial de ese documento y a la toma de medidas efectivas por parte de la EPA. El juez, no obstante admitir la difícil situación de la población de abejas y de los apicultores, entendió que las decisiones de política ambiental le corresponden a la EPA, y que el rol de los jueces se limita a la revisión judicial de las decisiones finales de la Agencia, lo cual no incluía el documento impugnado.

No obstante lo expuesto, la justicia estadounidense también cuenta con antecedentes favorables a los apicultores. En mayo de 2017, una Corte de Distrito de California (Estados Unidos), determinó que la Agencia de Protección Ambiental (EPA) había violado la Ley de Especies en Peligro de Extinción (Endangered Species Act, ESA) y la Ley Federal de Insecticidas, Fungicidas y Rodenticidas (Federal Insecticide, Fungicide, and Rodenticide Act, FIFRA) al aprobar y permitir la registración de 59 pesticidas neonicotinoides entre 2007 y 2012 (Case No. 13-cv-01266-MMC). Los demandantes alegaron que los plaguicidas en cuestión habían demostrado tener efectos adversos tanto en las abejas melíferas y otros polinizadores, como en otros animales, incluidas especies amenazadas y en peligro de extinción. Según la Corte, los demandantes tenían un interés económico que había sido afectado, en tanto esos pesticidas habían sido reconocidos por la EPA como "tóxicos para las abejas melíferas". 11

Algunos tribunales también han permitido a los apicultores organizar sus reclamos colectivamente. En febrero de 2018, el Tribunal Supremo de la Provincia de Québec (Canadá) autorizó a un grupo de apicultores a llevar adelante una acción de clase contra Bayer y Syngenta, los principales fabricantes de neonicotinoides de Québec (No: 500-06-000714-143). El apicultor Martineau, como representante de la clase, denunció un importante decrecimiento en la población de abejas que tuvo comienzo en el año 2006, con una inusual mortalidad y atrofia en abejas reinas y larvas, y huevos afectados por deshidratación.

El Tribunal Supremo consideró que todos los miembros de la clase sufrieron y sufren los daños causados por los neonicotinoides que son diseñados, fabricados, comercializados y distribuidos

\footnotetext{
${ }^{10}$ Es una guía para los inspectores federales, estatales y tribales que realizan inspecciones bajo la Ley Federal de Insecticidas, Fungicidas y Rodenticidas (FIFRA) como resultado de la muerte de abejas melíferas y otras abejas sociales.

${ }^{11}$ Para un abordaje más detallado véase Walker \& Wu (2017).
} 
Bailo, G.LLitigación sobre polinizadores (el derecho según las abejas)Derecho y Ciencias Sociales. Mayo-Octubre 2019. № 21. Pgs 263-283. ISNN 1852-2971. Instituto de Cultura Jurídica y Maestría en Sociología Jurídica. FCJ y S. UNLP

por los demandados en la Provincia de Québec. Por tal razón, la demanda se extendió a toda la clase, lo cual incluye a todas las personas en Québec que poseen o poseyeron abejas en el área afectada durante el período fijado para la clase (enero 2006 - febrero 2018). A partir de estas consideraciones, la Corte entendió que las principales cuestiones de hecho y derecho de esta acción deberán ser resueltas colectivamente, sin perjuicio de la prueba respecto de los daños que los apicultores sufrieron individualmente.

\section{La litigación sobre el sulfoxaflor}

No obstante lo expuesto, la litigación sobre insecticidas y polinizadores no se agota en los neonicotinoides. El 6 de mayo de 2013, la EPA (Estados Unidos) aprobó los primeros dos pesticidas que contenían sulfoxaflor, bajo las marcas "Transform” y "Closer”, de la corporación Dow Chemical.

La aparición en el mercado del sulfoxaflor provocó una controversia respecto a su clasificación. Para algunos expertos el sulfoxaflor es una cuarta generación de neonicotinoides (ver Cutler et al, 2013). En una posición contraria, la Corporación Dow Chemical lo describe como una nueva clase de insecticida que pertenece a las sulfoximinas, y que actúa sobre el sistema nervioso central de los insectos de forma distinta a los neonicotinoides.

El Comité de Acción contra la Resistencia a Insecticidas (IRAC) clasificó el sulfoxaflor como insecticida del grupo 4C, quedando separado de los neonicotinoides (grupo 4A), butenolides (grupo 4D) y mesoiónicos (grupo 4E). La Red de Acción en Plaguicidas, una coalición de organizaciones no gubernamentales, produjo un informe en el que critica la clasificación mencionada, por considerarla una maniobra para evitar las regulaciones existentes (véase Pesticide Action Network, 2016).

Los debates sobre el sulfoxaflor también han tenido repercusiones en la litigación. En septiembre de 2015, la Corte de Apelaciones del $9^{\circ}$ Circuito de California (Estados Unidos) resolvió anular el registro de sulfoxaflor realizado por la EPA y le ordenó a esta agencia la realización de estudios adicionales (806 F.3d 520). Para fundar su decisorio, la Corte de Apelaciones se apoyó en un dictamen del Panel de Asesoramiento Científico de la Ley Federal de Insecticidas, Fungicidas y Rodenticidas (FIFRA Scientific Advisory Panel). A partir de las consideraciones del dictamen del Panel, el tribunal sostuvo que la decisión de la EPA no estaba respaldada con pruebas sustanciales y que dejar subsistente el registro de sulfoxaflor en esas condiciones suponía un riesgo de daño ambiental. 
Los demandantes fueron apicultores y organizaciones de protección de abejas, que entendían que el registro del sulfoxaflor debía ser anulado porque los estudios demostraban que era altamente tóxico para las abejas. El Panel sostuvo que, dada la precariedad de las poblaciones de abejas, la decisión de dejar subsistente el registro de sulfoxaflor acarreaba mayor riesgo de daño ambiental que la decisión de anularlo. El fallo reconoce que las abejas son esenciales para la polinización y que en los últimos años han estado muriendo a tasas alarmantes. ${ }^{12}$

Sin embargo, el 14 de octubre de 2016 la EPA aprobó nuevamente la registración de los dos insecticidas a base de sulfoxaflor, alegando haber cumplimentado con los estudios adicionales que estipuló la sentencia analizada. La EPA definió al sulfoxaflor como una nueva clase de insecticidas, y lo consideró seguro para las abejas y los polinizadores.

La controversia sobre el estatus de este insecticida tuvo repercusión en los tribunales franceses. El 23 de noviembre de 2017, el tribunal administrativo de Niza (Francia) decidió suspender las autorizaciones que la Agence nationale de sécurité sanitaire de l'alimentation, de l'environnement et du travail (ANSES) había otorgado en septiembre del mismo año a los productos "Closer" y “Transform”, hasta que se determinara su legalidad (Casos $\mathrm{N}^{\circ} 1704688$ y N 1704690). El tribunal se apoyó en el principio precautorio, entendiendo que cuando hay incertidumbre sobre la existencia o alcance de los riesgos, las medidas de protección se pueden tomar sin esperar a que la realidad y gravedad de estos riesgos estén plenamente demostradas. También se expresó que, cuando es imposible determinar con certeza la existencia o el alcance del supuesto riesgo debido a la naturaleza insuficiente, inconclusa o imprecisa de los resultados de los estudios realizados, pero la probabilidad de daño real persiste, el principio de precaución justifica la adopción de medidas restrictivas.

\section{La litigación sobre el fipronil}

El fipronil es un insecticida de amplio espectro que pertenece a la familia de los fenilpirazoles. Los problemas asociados al uso del fipronil y la mortandad de abejas han sido particularmente graves en la República Oriental del Uruguay, dado que este insecticida causó una gran mortandad de abejas en 2009 cuando fue usado en las praderas deldepartamento de Flores. A partir de ello, "se prohibió el uso y la comercialización de la formulación líquida y en polvo de fipronil y se lo dejó únicamente como hormiguicida en una presentación granulada, y como curasemilla del arroz (este último uso se prohibió en 2014)” (Muñoz, 2017; cfr. Tapia, 2017).

\footnotetext{
${ }^{12}$ Para un estudio detallado de este caso véase Tabolsi (2016).
} 
El único uso autorizado del fipronil es en formulación granulada como hormiguicida y en el sector veterinario para combatir pulgas, garrapatas y ácaros. Sin embargo, el fipronil siguió asociado a la mortandad de abejas, como la que fue reportada en 2017 en el departamento de Salto (El Observador, 2017). ${ }^{13}$ Por estas razones, en Uruguay se ha discutido si el fipronil provoca la mortandad de abejas debido a su propia naturaleza tóxica o a su uso indebido.

En septiembre de 2014, la Suprema Corte de Justicia de la República Oriental del Uruguay resolvió condenar a los demandados por el daño que habían sufrido las colonias de abejas del actor, localizadas en el departamento de Flores (Nro: 827/2014). De acuerdo a la Suprema Corte, en diciembre de 2007 se produjo en este Departamento una situación que derivó en una grave afección a la producción apícola, y que tal situación se debió a la aplicación de un insecticida (no autorizado) grado 2 del principio activo fipronil con el nombre comercial "Fiproland FW 20". El fitosanitario, que no se encontraba autorizado por las autoridades correspondientes, había ingresado al país como hormiguicida y los demandados lo utilizaron para combatir la tucura, una especie que no estaba prevista. ${ }^{14}$

Los expertos que participaron del análisis de las muestras de miel y abejas concluyeron que existe un problema serio de intoxicación de colmenas a causa del fipronil. Agregaron que "en el caso de Flores existe una fuerte relación del problema de mortandad ocurrido con la aplicación de fipronil foliar, en praderas, para el control de Tucura”.

Para la Suprema Corte, las pruebas del expediente "permiten concluir con un grado de certeza razonable en que existió nexo de causalidad entre la fumigación realizada y la mortandad de las abejas propiedad del actor". Cada demandado fue condenado por una causal diferente. A la Unión Rural de Flores se la condenó porque sus técnicos aconsejaron la fumigación, sin formular advertencias especiales sobre la toxicidad para las abejas. Al segundo demandado se lo condenó por contratar el servicio de fumigación para su campo, lo que produjo finalmente los daños en las colmenas del actor.

En otro caso relacionado con el uso de fipronil en Uruguay, un Tribunal de Apelaciones consideró que no se daban los requisitos para responsabilizar al Estado (IUE: 0254-000704/2009. Nro: 0005000112/2014). Para decidir de esta manera, el Tribunal entendió que el producto había sido utilizado por productores agropecuarios a causa de un error técnico de las empresas de servicio y

\footnotetext{
${ }^{13}$ Se desprende de la noticia que el Ministerio de Agricultura, Ganadería y Pesca de Uruguay no pudo determinar el origen exacto de la contaminación por fipronil de las abejas, ya que el Ministerio alegó no haber encontrado rastros del insecticida en las plantaciones de la zona.

${ }^{14}$ De acuerdo a la sentencia, aunque se ignorase que el producto no estaba autorizado, debió procederse como lo indica la etiqueta de su envase que “...indica la toxicidad para las abejas, por lo que se debe retirar las colmenas antes de la aplicación a una distancia mínima aproximada de 4 kilómetros”.
} 
un asesoramiento inadecuado, y que el Ministerio de Ganadería, Agricultura y Pesca de Uruguay había autorizado y habilitado el uso en las condiciones que lucen en la etiqueta, por lo que la peligrosidad de su uso indebido estaba advertida. El Tribunal entendió que no se configuró la falta de servicio ya que se autorizó un producto, "si bien peligroso, pero estableciendo en forma clara que su uso se debía realizar en determinadas condiciones, con ciertos cuidados y requerimientos para no proferir daños a terceros".

El Tribunal también hizo algunas apreciaciones respecto de la relación causal entre el uso del producto y la mortandad de las abejas. Estimó que si bien "existen testimonios que refieren que aun bien aplicado el producto (sobre floración) ocasiona mortandad de abejas, pero ese extremo de importancia, si bien se oye sensato, debió ser demostrado mediante otro tipo de prueba, la idónea es la pericia sin dudas, prueba técnica y científica con respaldo académico que indique ello". También consideró que poco importa si se aplica el producto durante la floración, ya que "la colmena se supone debería estar distante al menos 4 kilómetros, tal como indica la etiqueta del producto, por lo que no se esperaría mortandad de abejas procediéndose de esa forma".El Tribunal tampoco ponderó las testimoniales que aseguraban que en épocas de sequía la abeja vuela a distancias mayores, "porque también se necesita un respaldo técnico y científico del punto que sólo puede ser aportado por un perito idóneo". 15

\section{La litigación sobre el glifosato y el ácido 2,4-diclorofenoxiacético}

El glifosato es un herbicida de amplio espectro, desarrollado para eliminar hierbas y arbustos.El ácido 2,4-diclorofenoxiacético (2,4 D) es un herbicida sistémico hormonal auxínico muy común, usado en el control de malezas de hoja ancha. Los problemas socioambientales asociados al monocultivo y al uso del glifosato son particularmente graves, especialmente en países como Argentina. Se ha dicho que "las prácticas de manejo como el monocultivo y el uso intensivo de agroquímicos reducen el número de especies de plantas, aves, insectos $\mathrm{y}$ otros grupos taxonómicos, a la vez que aumentan la abundancia relativa (dominancia) de pocas especies cultivadas y silvestres" (Garibaldi et al, 2017: 340).

Los tribunales argentinos han tenido ocasión de pronunciarse respecto a los efectos de estos herbicidas en la disminución de polinizadores en varias ocasiones, aunque con resultados discordantes.

\footnotetext{
${ }^{15}$ La conclusión del Tribunal es que no hay falta de servicio por parte de la Administración que se encuentre vinculada causalmente con el daño sufrido por los actores. A entender de los jueces, los responsables del daño fueron los aplicadores, quienes no tuvieron los cuidados debidos en el manejo de la cosa, desconociendo los términos en que fue autorizado el producto.
} 
En marzo de 2012, la Sala $3^{\circ}$ de la Cámara Civil y Comercial de Mar del Plata (Argentina), responsabilizó al dueño de un fundo por la muerte de las abejas del campo vecino (AP/JUR/95/2012). Las fumigaciones habían sido efectuadas con glifosato y con 2,4 D. La Cámara estimó que, aunque no existiera un grado de certeza absoluta acerca del motivo por el que murieron los insectos, "existen indicios que permiten llegar a la presunción de que tal mortandad fue causada por la fumigación efectuada en el campo lindero, al considerar que uno de los productos utilizados resulta ser tóxicos para las abejas, máxime si se tiene en cuenta que las abejas que por cuestiones climáticas no estuvieron expuestas a tales productos sobrevivieron”.

Uno de los aspectos de interés del fallo es el análisis que hace el tribunal del nexo causal entre la actividad de fumigación y la mortandad de las abejas. Al igual que en otros casos ya reseñados, estetribunal considera el vuelo de las abejas como un factor de particular importancia para trabajar en la delgada frontera delo probable y lo posible. ${ }^{16}$ Por un lado,el juez debía considerar que ambos productos actúan como herbicidas, que el glifosato está clasificado como no tóxico para las abejas y que el 2,4 D está clasificado como ligeramente tóxico para las abejas. Por otro lado, debía explorar los factores concretos quehabían desencadenado la mortandad de las abejas.De acuerdo al tribunal, "Si bien es cierto que no existe prueba en la causa que otorgue un grado de certeza absoluta acerca del motivo por el que murieron las abejas propiedad del actor, si existen indicios que permiten llegar a la presunción que tal mortandad fue causada por la fumigación efectuada por los codemandados".

Los elementos en los que se apoyaron los jueces para fundar esta conclusión fueron los siguientes: a) el carácter de "ligeramente tóxico" para abejas que tiene el producto 2,4 D; b) que las abejas que, por cuestiones geográficas, no estuvieron expuestas a tales productos sobrevivieron; c) que la no afectación de cinco de las setenta colmenas que se encontraban en el sector del campo donde se efectúo la fumigación se debió a que estas se encontraban hostigadas por el viento sur y por lo tanto las abejas no salieron de ellas en el momento en que se estaba fumigando; d) que en general las abejas no realizan vuelos en las condiciones climáticas descriptas en la causa, pero la ausencia total de nubes finalmente alentó a las abejas a salir a trabajar.

Una mirada diferente sobre el problema causal puede encontrarse en un fallo de la Suprema Corte de Justicia de la Provincia de Buenos Aires de junio de 2004, que confirmó un fallo de

\footnotetext{
${ }^{16}$ Respecto a la idea de posibilidad, de acuerdo al fallo, el dictamen pericial del Ingeniero Agrónomo interviniente expresa lo siguiente: "Digo que, en época invernal, con las condiciones climáticas del día 14/7/2006 en particular, dependiendo del volumen de líquido pulverizado por unidad de superficie, del tipo y calidad de producto utilizado y en particular de la distancia al apiario digo que "es posible" que parte de las obreras que salieran a trabajar no llegaran a la colmena y murieran en el trayecto...".
} 
Cámara,que a su vez confirmaba la sentencia de primera instancia que había rechazado la demanda de daños y perjuicios (Causa Ac. 87.410, Acuerdo 2078).En el fallo de Cámara, cuyos considerandos la Corte hace suyos, se sostuvo que el rechazo de la demanda debía fundarse en la falta de demostración de "la relación de causalidad adecuada entre la muerte de las abejas y la conducta desplegada por los demandados antes que en la falta de acreditación del daño o su extensión".

De acuerdo a la Cámara, la carga de la relación de causalidad entre el riesgo o vicio de la cosa o el hecho humano culposo o doloso y el daño pesaba sobre el actor que reclamaba el resarcimiento de los daños sufridos.La existencia de múltiples causas de mortandad fue interpretada como falta de prueba de la relación causal. ${ }^{17}$ Por ello, y de acuerdo a la lógica de este fallo, "la causalidad adecuada supone certeza y no probabilidad o mera posibilidad de que del hecho o cosa haya derivado el daño y que en la especie no se sabe por qué se murieron las abejas y así como tampoco si el producto utilizado en la ocasión para fumigar era idóneo para producir tal resultado".

En marzo de 2016, la Sala H de la Cámara Nacional de Apelaciones en Civil (Argentina), condenó al arrendatario de un predio lindante -que contrató el servicio de fumigación aérea con glifosato- a resarcir al propietario de un colmenar, por los daños y perjuicios derivados de la muerte de las abejas y la consecuente pérdida de producción de miel (MJ-JU-M-97767-AR).

La Cámara atribuyó responsabilidad a la arrendataria del predio lindante al del actor por el incorrecto uso del producto utilizado para la fumigación que hizo el servicio contratado. El tribunal entendió que no se configuraba la eximente de hecho de un tercero, porque la empresa de fumigaciones había sido contratada por el explotador del predio. En ese sentido, se encuadró la responsabilidad como objetiva.De acuerdo al tribunal, "el daño que se infiere in re ipsa, es aquel que no tiene otra explicación. Si se produjo la fumigación aérea, y a posteriori aparecieron las abejas muertas es correcto el razonamiento del sentenciante de grado de acudir a indicios y presunciones".

No obstante ello, la Cámara aceptó la excepción de falta de legitimaciónpasiva opuesta por Monsanto, porque "la actora no demostró fehacientemente que el producto fabricado por dicha empresa haya sido el utilizado en la fumigación que causó el daño, máxime cuando hay muchas

\footnotetext{
${ }^{17}$ La Corte expresa, retomando los argumentos de la Cámara, que “... el reclamante no había demostrado cuál había sido la causa de mortandad de las abejas de entre las múltiples posibles indicadas por el perito (v. fs. 210/211). Así lo reconoce el actor al absolver posiciones a fs. 98 -respuesta a la primera ampliación-. Tampoco acreditó que los demandados al fumigar hayan agregado a los herbicidas preemergentes -no dañinos-, productos piretroides o insecticidas que sí tienen tal capacidad dañadora (circunstancia ésta desconocida por los accionados- ver fs. 93 respuesta 3; fs. 95 respuesta 2- sin que ninguno de los testimonios aportados haya ido más allá de que en el campo vecino se fumigó...".
} 
otras empresas que lo fabrican y de las pruebas periciales surge que la causa de la muerte de las abejas fue el mal uso del producto".

Para fundar esta decisión, la Cámara emplea una analogía que adelanta la concepción de los jueces respecto a la manera de interpretar las relaciones entre derecho y tecnología. Dice que demandar a Monsanto "es como si en una demanda por los daños resultantes de un homicidio, se demandara también al fabricante de la pistola utilizada”.Por esta razón, en este caso la presencia de múltiples factores causales operó como un límite al conocimiento judicial. Según el tribunal, la prueba colectada "pone bien a las claras la existencia de múltiples factores que, más allá de las bondades del producto aplicado, pudieron incidir en la muerte de las abejas. Tales factores adicionales, en ausencia de mejor prueba concreta, sólo autorizan a conjeturar”.

\section{La litigación sobre el dimetoato}

El dimetoato es un insecticida y acaricida organofosforado, altamente tóxico para abejas y aves. En febrero de 2006, un Juzgado de Chos Malal (Neuquén, Argentina) condenó a la Provincia de Neuquén por los daños y perjuicios sufridos a raíz de la muerte de abejas, producida a consecuencia de la fumigación con dimetoato efectuada por una Estación Agrozootécnica en las chacras donde se situaban los colmenares del actor (AR/JUR/199/2006).

De acuerdo a las constancias de la causa, la demandada propició la fumigación proveyendo el vehículo, el personal y el insecticida al productor agropecuario que se lo solicitó, sin dar cumplimiento a las previsiones de la ley local $\mathrm{n}^{\circ}$ 1796, “que prohíbe la pulverización con agroquímicos sobre colmenares $\mathrm{y}$, en caso de tratamiento con pasturas, obliga a poner en conocimiento con suficiente antelación a los productores apícolas a los efectos de que estos instrumenten las medidas preventivas convenientes".

El juzgado entendió que no se dio previo aviso suficiente de la realización de la fumigación, que no se advirtió la peligrosidad del plaguicida, que el plaguicida era una cosa riesgosa que la tornaba inapropiada para una utilización inocua, que el demandado no previó las consecuencias de los actos que ejecutó, al no hacer una adecuación objetiva entre el hecho y el evento según las reglas de la experiencia y el normal cálculo de probabilidades. ${ }^{18}$

\section{Algunas conclusiones}

Como se ha visto a lo largo del trabajo, la presencia de polinizadores es esencial para la biodiversidad y la producción de alimentos. Estas especies, particularmente a través de sus vuelos,

\footnotetext{
${ }^{18}$ De acuerdo a la pericia producida por el actor, la muerte de las abejas se produjo por el pesticida dimetoato, aplicado sobre cuadros de alfalfa vecinos inmediatos a la localización de las colmenas.
} 
producen su propia narrativa sobre las relaciones que existen entre los distintos factores de estrés a los que están sometidos y su mortandad. Por ello, hemos intentado reconstruir unalínea jurisprudencial,no necesariamente uniforme o consolidada, que demuestra un ligero viraje de la litigación clásica sobre las abejas melíferas (centrada en la propiedad y la responsabilidad por daños) a la litigación sobre polinizadores (centrada en la protección de la biodiversidad y la alimentación).

No obstante ello, esta litigación sobre polinizadores ha tenido problemas para construir una legitimación colectiva que supere la consideración de las abejas como un mero recurso natural con valor económico y para lidiar con la insuficiencia del conocimiento existente en la materia. Se han dado avances en materia de legitimación colectiva en Francia y en Canadá. También hay progresos en materia de revisión de reglamentos administrativos en el ámbito de la Unión Europea, en Francia y en Estados Unidos. El principio precautorio ha sido utilizado en el Tribunal General de la Unión Europea y en varios tribunales en Francia. En Estados Unidos, los tribunales han preferido remitirse a leyes de conservación de especies y leyes que regulan el uso de insecticidas. Los tribunales de Argentina y Uruguay han trabajado los casos de polinizadores desde las herramientas generales de la responsabilidad civil, especialmente desdela prueba de la relación causal y la cuantificación de los daños.

Por otra parte, la litigación sobre polinizadores se ha concentrado casi exclusivamente en el problema de los pesticidas y no ha incursionado significativamente en otras causas de mortandad (fragmentación del paisaje, monocultivo, cambio climático, especies invasoras). De la misma manera, que los apicultores sean los principales promotores de esta litigación explica que casi todas las demandas se concentren en la protección dedistintas especies de abejas melíferas, lo que excluye a otros tipos de polinizadorescomo las polillas,mariposas, murciélagos,escarabajos, moscas, algunas aves, entre otros.

\section{Referencias bibliográficas}

Baines, D., Wilton, E., Pawluk, A., Gorter, M., \& Chomistek, N. (2017). Neonicotinoids act like endocrine disrupting chemicals in newly-emerged bees and winter bees.Scientific Reports, 7(1), 10979.

Cutler, P., Slater, R., Edmunds, A. J., Maienfisch, P., Hall, R. G., Earley, F. G., et al. (2013). Investigating the mode of action of sulfoxaflor: a fourth $\square$ generation neonicotinoid. Pest management science,69(5), 607-619. 
Bailo, G.LLitigación sobre polinizadores (el derecho según las abejas)Derecho y Ciencias Sociales. Mayo-Octubre 2019 . № 21. Pgs 263-283. ISNN 1852-2971. Instituto de Cultura Jurídica y Maestría en Sociología Jurídica. FCJ y S. UNLP

Doutt, R. L. (1959). The Case of the Trespassing Bees. Bulletin of the ESA, 5(3), 93-97.

Garibaldi, L. A., Aguiar, S., Aizen, M. A., Morales, C. L., \& Sáez, A. (2017). ¿Diversidad o dominancia en la producción de alimentos? El caso de los polinizadores.Ecología Austral, 27(3), 340-347.

Gould, J. (2015). Meet our prime pollinators. Nature, 521(7552), S48-S48.

Goulson, D., Nicholls, E., Botías, C., \& Rotheray, E. L. (2015). Bee declines driven by combined stress from parasites, pesticides, and lack of flowers.Science,347(6229), 1255957.

Jacobs, M. (1999). Anklagemyndigheden v. Ditlev Bluhme, Case C $\square 67 / 97$ (Danish Bees case). Review of European Community \& International Environmental Law,8(2), 220-223.

Jiménez, A. G. (2018). La protección jurídica y administrativa de las abejas.Actualidad Jurídica Ambiental, (80), 39-72.

Kessler, S. C., Tiedeken, E. J., Simcock, K. L., Derveau, S., Mitchell, J., Softley, S., et al. (2015). Bees prefer foods containing neonicotinoid pesticides.Nature,521(7550), 74.

Mayr, E (2016). Así es la biología. Madrid: Debate.

Ortiz García, M. (2014). La primavera gris: sobre el declive de las abejas.Revista Vasca de Administración Pública. Herri-Arduralaritzako Euskal Aldizkaria, (99), 2261-2287.

Pimentel, D., Acquay, H., Biltonen, M., Rice, P., Silva, M., Nelson, J., et al.(1992). Environmental and economic costs of pesticide use.BioScience,42(10), 750-760.

Potts, S. G., Biesmeijer, J. C., Kremen, C., Neumann, P., Schweiger, O., \& Kunin, W. E. (2010). Global pollinator declines: trends, impacts and drivers.Trends in ecology \& evolution, 25(6), 345353.

Tabolsi, A. (2016). Pollinator Stewardship Council v. EPA and the Duty to Research FIFRA Applications.Ecology LQ,43, 503.

Von Frisch, K. (1955). The dancing bees. New York: Harcourt, Brace.

Von Frisch, K. (1974). Decoding the language of the bee.Science,185(4152), 663-668.

Walker, L., \& Wu, S. (2017). Pollinators and Pesticides. En International Farm Animal, Wildlife and Food Safety Law(pp. 495-513). Berlín: Springer, Cham.

Williams, G. R., Troxler, A., Retschnig, G., Roth, K., Yañez, O., Shutler, D., et al. (2015). Neonicotinoid pesticides severely affect honey bee queens.Scientific reports,5, 14621. 
Bailo, G.LLitigación sobre polinizadores (el derecho según las abejas)Derecho y Ciencias Sociales. Mayo-Octubre 2019 . № 21. Pgs 263-283. ISNN 1852-2971. Instituto de Cultura Jurídica y Maestría en Sociología Jurídica. FCJ y S. UNLP

\section{Noticias citadas}

Benjamin, A. (23 de mayo de 2008). Pesticides: Germany bans chemicals linked to honeybee devastation. The Guardian. Recuperado de: https://www.theguardian.com/environment/2008/may/23/wildlife.endangeredspecies

El Observador (28 de septiembre de 2017). Un insecticida prohibido causó la muerte de abejas en Salto. Recuperado de: https://www.elobservador.com.uy/nota/un-insecticida-prohibido-causo-la$\underline{\text { muerte-de-abejas-en-salto-2017928500 }}$

Muñoz, A. (28 de septiembre de 2017). MGAP constató que las abejas fueron envenenadas con fipronil, con una presentación prohibida en Uruguay desde 2009. La Diaria. Recuperado de: https://ladiaria.com.uy/articulo/2017/9/mgap-constato-que-las-abejas-fueron-envenenadas-confipronil-con-una-presentacion-prohibida-en-uruguay-desde-2009/

Tapia, C. (29 de octubre de 2017). El temor a que dejen de zumbar. El País. Recuperado de: https://www.elpais.com.uy/que-pasa/temor-dejen-zumbar.html

\section{Documentosy normativa}

Asamblea General de la Organización de las Naciones Unidas (20 de diciembre de 2017). Día Mundial de las Abejas. A/RES/72/211. Disponible en: https://undocs.org/es/A/RES/72/211

Autoridad Europea de Seguridad Alimentaria (28 de febrero de 2018). Q\&A: Conclusions on neonicotinoids 2018. Disponible en: https://www.efsa.europa.eu/sites/default/files/news/180228QA-Neonics.pdf

Comisión Europea. Reglamento de Ejecución (UE) no 485/2013 de la Comisión. Disponible en: https:/eur-lex.europa.eu/LexUriServ/LexUriServ.do?uri=OJ:L:2013:139:0012:0026:ES:PDF

Comité Scientifique et Technique de l'Etude Multifactorielle des Troubles des Abeilles (18 de septiembre de 2003). Imidaclopride utilisé en enrobage de semences (Gaucho®) et troubles des abeilles Rapport final. Disponible en: $\underline{\mathrm{http}} / / /$ controverses.sciencespo.fr/archive/pesticides/rapportfin.pdf

Convenio Diversidad Biológica (6 de abril de 2018). Conservation and sustainable use of pollinators. CBD/SBSTTA/22/10. Disponible en: https://www.cbd.int/doc/c/ca37/8073/bcc8bf627c0d00bdb6f4ab9a/sbstta-22-10-en.pdf

Francia. LOI n ${ }^{\circ} 2016-1087$ du 8 août 2016 pour la reconquête de la biodiversité, de la nature et des paysages. NOR : DEVL1400720L. 
Italia. Decreto ministeriale 17 settembre 2008. Sospensione cautelativa dell'autorizzazione di impiego per la concia di sementi, dei prodotti fitosanitari contenenti le sostanze attive clothianidin, thiamethoxam, imidacloprid e fipronil. Gazzetta Ufficiale n. 221 del 20.09.2008.

Pesticide Action Network (septiembre de 2016). Sulfoxaflor and flupyradifurone: Neonicotinoids or not? Disponible en: https://www.pan-europe.info/sites/paneurope.info/files/public/resources/factsheets/201609\%20Factsheet $\% 20$ What $\% 20$ is $\% 20 \mathrm{a} \% 20$ neoni cotinoid_Flupyradifurone_Sulfoxaflor_EN_PAN\%20Europe.pdf

República Federal de Alemania (13 de mayo de 2008). Background information on endemic damage to bees in South Germany. Federal Office of Consumer Protection and Food Safety.

Disponible en: https://www.bvl.bund.de/EN/08_PresseInfothek_engl/01_Presse_und_Hintergrundinformationen/ $\underline{2008 \quad 0513 \text { hi_Bienensterben en.html }}$

Rete Rurale Nazionale (s.f.). Sospensione impiego dei neonicotinoidi per la concia delle sementi. Disponible en: https://www.reterurale.it/flex/cm/pages/ServeBLOB.php/L/IT/IDPagina/815

Tribunal Permanente de los Pueblos (diciembre de 2011). Session on Agrochemical Transnational Corporations. Disponible en: http://permanentpeoplestribunal.org/wpcontent/uploads/2011/12/TPP_Bangalore3Dec2011.pdf

United States Environmental Protection Agency (s.f.). Decision to Register the Insecticide Sulfoxaflor with Limited Uses and Pollinator Protective Requirements. Disponible en: https://www.epa.gov/ingredients-used-pesticide-products/decision-register-insecticide-sulfoxaflorlimited-uses-and

United States Environmental Protection Agency (9 de mayo de 2013). Guidance for Inspecting Alleged Cases of Pesticide-Related Bee Incidents. Disponible en: https://www.epa.gov/sites/production/files/2013-09/documents/bee-inspection-guide.pdf

\section{Jurisprudencia citada}

Argentina. 03/03/2016. Cámara Nacional de Apelaciones en lo Civil Sala H. Zengaro Juan José c/ Monsanto Argentina S.A.I.C. y otros s/ daños y perjuicios. Cita: MJ-JU-M-97767-AR | MJJ97767 | MJJ97767.

Argentina. 08/07/2013. Cámara de Apelaciones en lo Civil y Comercial de Mar del Plata, sala I. Romero, Horacio Miguel y otra c. Fidanza, Juan Roberto y otros s/ daños y perjuicios. $\mathrm{AR} / \mathrm{JUR} / 48408 / 2013$. 
Bailo, G.LLitigación sobre polinizadores (el derecho según las abejas)Derecho y Ciencias Sociales. Mayo-Octubre 2019 . № 21. Pgs 263-283. ISNN 1852-2971. Instituto de Cultura Jurídica y Maestría en Sociología Jurídica. FCJ y S. UNLP

Argentina. 16/03/2012. Cámara Civil y Comercial Mar del Plata sala 3a . Errobidart, Juan Pablo v. Tirso Bilbao - Bilbao César Sociedad de Hecho. AP/JUR/95/2012.

Argentina. 20/02/2006. Juzgado de 1a Instancia en lo Civil, Comercial, Laboral, y de Minería de la V Circunscripción Judicial, Chos Malal. Rolny, Eleodoro D. c. Provincia del Neuquén. AR/JUR/199/2006.

Argentina. 09/06/2004. Suprema Corte de Justicia de la Provincia de Buenos Aires. "Aguiar, Pablo Daniel contra Tittarelli, Aníbal y otro. Daños y perjuicios". Causa Ac. 87.410, Acuerdo 2078 .

Canadá. 20/02/2018. Superior Court. Province of Québec. District of Montréal. Martineau v. Bayer and Syngenta. No: 500-06-000714-143.

Francia. 09/12/2008. Cour d'Appels d'Angers 1ère Chambre A. Michel Ligne v Cooperative agricole Terrena. Affaire $n^{\circ}$ 07/01836.

Francia. 23/11/2017. Tribunal Administratif de Nice. Association Generations Futures au nom du Peuple Français. № 1704688.

Francia. 23/11/2017. Tribunal Administratif de Nice. Association Generations Futures au nom du Peuple Français. № 1704690.

República Oriental del Uruguay. 11/06/2014. Tribunal Apelaciones Civil $2^{\circ} \mathrm{T}^{\mathrm{o}}$. López Cedrés Daniel y Otros c/ Ministerio de Agricultura, Ganadería y Pesca. Daños y perjuicios, IUE: 0254000704/2009. Nro: 0005-000112/2014.

República Oriental del Uruguay. 24/09/2014. Suprema Corte de Justicia. Vignolo Cutinella, Nestor c/ Cuevas Caceres, Arturo y Otros - Cobro de Pesos - Daños y Perjuicios - Casación, IUE: 254-695/2009. Nro: 827/2014.

Tribunal de Justicia de la Unión Europea. 03/12/1998. Anklagemyndigheden v. Ditlev Bluhme. Asunto C-67/97. ECLI:EU:C:1998:584.

Tribunal General de la Unión Europea. 17/05/2018. Bayer CropScience AG y otros contra Comisión Europea. Sentencias en los asuntos acumulados T-429/13 Bayer CropScience AG y otros / Comisión y T-451/13 Syngenta Crop Protection AG y otros / Comisión, y en el asunto T584/13 BASF Agro BV y otros / Comisión. ECLI:EU:T:2018:280.

United States. 08/05/2017. District Court, N.D. California. Ellis v. Housenger. Case No. 13-cv01266-MMC. 252 F.Supp.3d 800. 
Bailo, G.LLitigación sobre polinizadores (el derecho según las abejas)Derecho y Ciencias Sociales. Mayo-Octubre 2019. № 21. Pgs 263-283. ISNN 1852-2971. Instituto de Cultura Jurídica y Maestría en Sociología Jurídica. FCJ y S. UNLP

United States. 10/09/2015. Court of Appeals for the 9th Circuit of California. Pollinator Stewardship Council v. U.S.E.P.A. Reference: 13-72346. 806 F.3d 520.

United States. 21/11/2016. District Court, N.D. California. Anderson v. McCarthy. Case No. 3:16cv-00068-WHA. 